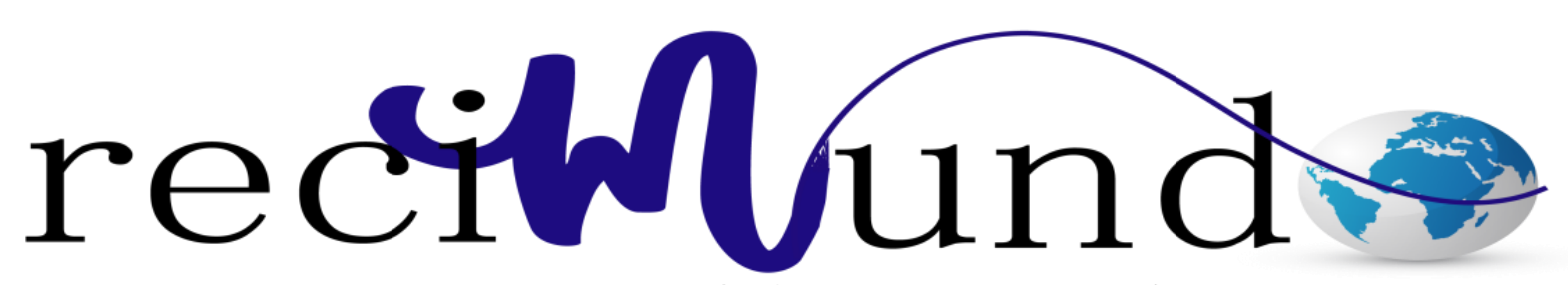

Revista Científica Mundo de la Investigación y el Conocimiento

Roberto Johan Barragán Monrroy a ; Johana Narcisa Ormaza Varela ${ }^{\text {b; }}$ Gissela Eloísa Vargas Cedeño ${ }^{c}$; Gabriela Verónica Estrella Bravo ${ }^{d}$

Derechos de la naturaleza frente a los delitos ambientales provocados por las actividades antropogénicas en la provincia de Los Ríos

Rights of nature against environmental crimes caused by anthropogenic activities in the province of Los Ríos

Revista Científica Mundo de la Investigación y el Conocimiento. Vol. 3 núm.3. Esp., noviembre, ISSN: 2588-073X, 2019, pp. 481-497

DOI: $10.26820 /$ recimundo/3.(3.Esp ).noviembre.2019.481-497

URL: http://recimundo.com/index.php/es/article/view/619

Código UNESCO: 5605 Legislación y Leyes Nacionales

Tipo de Investigación: Artículo de Revisión

(C) RECIMUNDO; Editorial Saberes del Conocimiento, 2019

Recibido: 15/09/2019

Aceptado: 23/10/2019

Publicado: 30/11/2019

Correspondencia: robertobarraganm81@gmail.com

\footnotetext{
a. Ingeniero en Gestión Ambiental; Investigador Independiente; Guayaquil, Ecuador; robertobarraganm81@ gmail.com

b. Ingeniera en Gestión Ambiental; Universidad Técnica Estatal de Quevedo; Guayaquil, Ecuador; johana.ormaza2014@uteq.edu.ec

c. Investigadora Independiente; Guayaquil, Ecuador; gissela.vargas2013@uteq.edu.ec

d. Ingeniera en Gestión Ambiental; Universidad Técnica Estatal de Quevedo; Guayaquil, Ecuador; gabriela.estrella2014@uteq.edu.ec
} 


\section{Derechos de la naturaleza frente a los delitos ambientales provocados por las actividades antropogénicas en la provincia de Los Ríos}

Vol. 3, núm. 3 Esp., (2019)

Roberto Johan Barragán Monrroy; Johana Narcisa Ormaza Varela; Gissela Eloísa Vargas

Cedeño; Gabriela Verónica Estrella Bravo

\section{RESUMEN}

La determinación de la naturaleza como sujeto de derechos, responde a la teoría ecocéntrica, la cual coloca al ambiente y a la naturaleza como el eje central de las cuestiones ambientales. Esta corriente ha influenciado instrumentos tales como la Carta de la Naturaleza de la Naciones Unidas de 1982. La nueva Constitución de Ecuador, aprobada en septiembre de 2008, presenta por primera vez en América Latina los derechos propios de la Naturaleza. En ella se reconoce expresamente a la naturaleza como sujeto de derecho y ha pretendido generar un cambio conceptual sustancial respecto a varios temas como el régimen de desarrollo y la inclusión del buen vivir o sumak kawsay como concepto orientador de la vida. Los delitos ambientales se definen como un crimen contra el medio ambiente que es sancionado por la legislación medioambiental. Es una infracción contra la legislación medioambiental, cuya sanción judicial está clasificada en la categoría de crimen. Las actividades antropogénicas son aquellas relacionadas con la influencia del hombre en la naturaleza. El término antropogénico se ha utilizado con énfasis sobre la cantidad de dióxido de carbono que se encuentra en la atmósfera, producida por las actividades humanas y con un gran efecto sobre el cambio climático contemporáneo. Existen diferentes tipos: la atmosférica, hídrica, de los suelos, radioactiva, visual, lumínica, acústica o por basura. La crisis ambiental en muchos casos es severa, no existen métodos detallados para medirla cabalmente, hay evidencia suficiente que afecta al desarrollo de países y sociedades, tanto ricos, como pobres. La metodología usada es descriptiva, con un enfoque documental, es decir, revisar fuentes disponibles en la red, como google académico, con contenido oportuno y relevante desde el punto de vista científico que enriquezca el análisis del tema planteado en este artículo.

Palabras claves: Buen vivir; Delitos ambientales; Actividades antropogénicas; Tala; Contaminación de agua; Tráfico de animales. 


\title{
Derechos de la naturaleza frente a los delitos ambientales provocados por las actividades antropogénicas en la provincia de Los Ríos
} Vol. 3, núm. 3 Esp., (2019) Roberto Johan Barragán Monrroy; Johana Narcisa Ormaza Varela; Gissela Eloísa Vargas

Cedeño; Gabriela Verónica Estrella Bravo

\begin{abstract}
The determination of nature as a subject of rights, responds to the ecocentric theory, which places the environment and nature as the central axis of environmental issues. This current has influenced instruments such as the United Nations Nature Charter of 1982. The new Constitution of Ecuador, approved in September 2008, presents for the first time in Latin America the rights of Nature itself. In it, nature is expressly recognized as a subject of law and has sought to generate a substantial conceptual change regarding various issues such as the development regime and the inclusion of good living or sumak kawsay as a guiding concept of life. Environmental crimes are defined as a crime against the environment that is sanctioned by environmental legislation. It is an infraction against environmental legislation, whose judicial sanction is classified in the crime category. Anthropogenic activities are those related to the influence of man in nature. The term anthropogenic has been used with emphasis on the amount of carbon dioxide in the atmosphere, produced by human activities and with a great effect on contemporary climate change. There are different types: atmospheric, water, soil, radioactive, visual, light, and acoustic or garbage. The environmental crisis in many cases is severe, there are no detailed methods to measure it fully, and there is sufficient evidence that affects the development of countries and societies, both rich and poor. The methodology used is descriptive, with a documentary approach that is, reviewing sources available on the web, such as google scholar, with timely and scientifically relevant content that enriches the analysis of the topic raised in this article.
\end{abstract}

Keywords: Good living; Environmental crimes; Anthropogenic activities; Logging; Water pollution; Animal trafficking. 


\section{Derechos de la naturaleza frente a los delitos ambientales provocados por las actividades antropogénicas en la provincia de Los Ríos}

Vol. 3, núm. 3 Esp., (2019)

Roberto Johan Barragán Monrroy; Johana Narcisa Ormaza Varela; Gissela Eloísa Vargas

Cedeño; Gabriela Verónica Estrella Bravo

\section{Introducción.}

La preocupación por el medio ambiente prevalece y motiva a poner en vigencia normas tanto administrativas como penales que impidan su deterioro y mermen sustentabilidad. El avance del desarrollo industrial y tecnológico que lo ha afectado grandemente, y de modo irremediable, en muchos casos. Aceptada la necesaria intervención del Derecho y la protección jurídica del medio ambiente, es evidente la necesidad de contar con una coacción jurídica que evite la destrucción progresiva del medio ambiente, porque éste representa el medio del crecimiento económico en el que se ha de mantener un indispensable equilibrio, que, de romperse, terminaría con la propia existencia del hombre por la destrucción de la naturaleza y el entorno en el que vive.

En el último reporte global del Convenio de Diversidad Biológica se concluye que una de las mayores causas de la pérdida de biodiversidad está dada por las presiones vinculadas a la agricultura, que abarcan $70 \%$ de la pérdida estimada de la biodiversidad terrestre. Por ello, como punto crucial, recomienda reorientar las tendencias de los sistemas alimentarios, buscando una producción sostenible y restaurando los servicios ecosistémicos en paisajes agroecológicos (CEPAL, 2017) "La pérdida de biodiversidad es rápida y continua. Durante los últimos 50 años, los seres humanos hemos cambiado los ecosistemas más rápida y extensamente que en cualquier otro período comparable de la historia de la humanidad. Las causas directas de la pérdida de biodiversidad no muestran señales de disminución “.

No es una simple alarma o exageración por parte de ecologistas agrupados en distintas organizaciones en todo el mundo. Es que la destrucción del medio ambiente puede tener una 


\section{Derechos de la naturaleza frente a los delitos ambientales provocados por las actividades antropogénicas en la provincia de Los Ríos}

Vol. 3, núm. 3 Esp., (2019) Roberto Johan Barragán Monrroy; Johana Narcisa Ormaza Varela; Gissela Eloísa Vargas Cedeño; Gabriela Verónica Estrella Bravo

dimensión tal que amenace realmente el futuro del hombre sobre la tierra. En este punto, hay que tomar conciencia de que la destrucción del bien jurídico ambiente es obra no sólo de vándalos sino del ciudadano común Lo realmente grave es que es el ciudadano común y corriente el que al estar preocupado por otras metas.

La nueva Constitución de Ecuador, aprobada en septiembre de 2008, presenta por primera vez en América Latina los derechos propios de la Naturaleza. Este giro al biocentrismo, entendido como el reconocimiento de valores intrínsecos en el ambiente, es el hecho más significativo en la ecología política Latinoamericana de los últimos años desarrollo y la inclusión del buen vivir o sumak kawsay como concepto orientador de la vida. La Constitución Ecuatoriana determina que la naturaleza será sujeto de aquellos derechos que le reconozca la Constitución, es decir, que por un lado se entregó derechos subjetivos a la naturaleza, reconociendo el valor intrínseco de la naturaleza independientemente de su utilidad y por otro lado, se estableció una reserva constitucional para el establecimiento de estos derechos.

Conscientes de que la protección jurídica del medio ambiente es hoy una necesidad universalmente reconocida, que se trata es de conciliar el desarrollo con el medio ambiente, reconociendo que es esta una tarea vital de las personas sensatas que viven en esta época de la historia de la humanidad, es conveniente desarrollar este y otros aspectos durante este artículo, también conocer las penas y sanciones que estos delitos acarrean en Ecuador. 


\section{Derechos de la naturaleza frente a los delitos ambientales provocados por las actividades antropogénicas en la provincia de Los Ríos}

Vol. 3, núm. 3 Esp., (2019)

Roberto Johan Barragán Monrroy; Johana Narcisa Ormaza Varela; Gissela Eloísa Vargas

Cedeño; Gabriela Verónica Estrella Bravo

\section{Metodología.}

Esta investigación está dirigida al estudio de "Derechos de la naturaleza frente a los delitos ambientales provocados por las actividades antropogénicas en la provincia de Los Ríos”. Para realizarlo se usó una metodología tipo descriptiva, con un enfoque documental, es decir, revisar fuentes disponibles en la red, como google académico, con contenido oportuno y relevante desde el punto de vista científico para dar respuesta a lo tratado en el presente artículo y que sirvan de inspiración para realizar otros proyectos. Las mismas pueden ser consultadas al final, en la bibliografía.

\section{Resultados.}

La concepción de la naturaleza como objeto de la relación jurídica tiene su fundamento en la teoría antropocéntrica, la cual sigue la posición tradicional jurídica romanista. Por esta razón, el derecho ambiental ha concebido al ser humano como el centro del universo y a la naturaleza como un ente que le permite satisfacer sus necesidades, por este motivo la protege; en consecuencia, el ser humano es sujeto de derechos y el ambiente objeto.

La determinación de la naturaleza como sujeto de derechos, por su parte, responde a la teoría ecocéntrica, la cual coloca al ambiente y a la naturaleza como el eje central de las cuestiones ambientales. Esta corriente ha influenciado instrumentos tales como (Bedón, Abril 2017) "la Carta de la Naturaleza de la Naciones Unidas de 1982 en la cual se establece que la especie humana es parte de la naturaleza y la vida depende del funcionamiento ininterrumpido de 


\section{Derechos de la naturaleza frente a los delitos ambientales provocados por las actividades antropogénicas en la provincia de Los Ríos}

Vol. 3, núm. 3 Esp., (2019) Roberto Johan Barragán Monrroy; Johana Narcisa Ormaza Varela; Gissela Eloísa Vargas Cedeño; Gabriela Verónica Estrella Bravo

los sistemas naturales; señala además que toda forma de vida es única y merece ser respetada, cualquiera sea su utilidad para el ser humano" (103).

La teoría de considerar como sujeto a la naturaleza fue desarrollada inicialmente por Christopher D. Stone autor estadounidense que, gracias a un fallo judicial, escribió un ensayo en el que sostiene que los árboles Secuoyas debían ser preservados por sí mismos ya que son sujetos de derechos. Esta postura, fue redefinida por el mismo autor, al señalar que para proteger a la naturaleza antes que reconocer su calidad de sujeto de derechos es necesario imponer deberes a los seres humanos. El profesor Stone planteó: los árboles debían tener derecho a representación legal y en los casos en que fueran objeto de daño también deberían tener derecho a la reparación (Martínez, 2019).

La nueva Constitución de Ecuador, aprobada en septiembre de 2008, presenta por primera vez en América Latina los derechos propios de la Naturaleza. Este giro al biocentrismo, entendido como el reconocimiento de valores intrínsecos en el ambiente, es el hecho más significativo en la ecología política Latinoamericana de los últimos años (Gudynas, 2009). En ella se reconoce expresamente a la naturaleza como sujeto de derecho y ha pretendido generar un cambio conceptual sustancial respecto a varios temas como el régimen de desarrollo y la inclusión del buen vivir o sumak kawsay como concepto orientador de la vida. Se entiende el buen vivir como una categoría simbólica que denota, un conjunto de valores que dan sentido a la existencia en el plano individual y colectivo. Vida en armonía que conjuga la relación con el entorno natural, la tierra sin mal y con la cultura o sabiduría de los ancestros. Pues resulta evidente que para el efectivo cumplimiento de los derechos de la naturaleza se requiérela 


\section{Derechos de la naturaleza frente a los delitos ambientales provocados por las actividades antropogénicas en la provincia de Los Ríos}

Vol. 3, núm. 3 Esp., (2019)

Roberto Johan Barragán Monrroy; Johana Narcisa Ormaza Varela; Gissela Eloísa Vargas

Cedeño; Gabriela Verónica Estrella Bravo

coexistencia de la armonía de los seres humanos con la naturaleza, que es exactamente el objetico del buen vivir.

La Constitución Ecuatoriana determina que la naturaleza será sujeto de aquellos derechos que le reconozca la Constitución, es decir, que por un lado se entregó derechos subjetivos a la naturaleza, reconociendo el valor intrínseco de la naturaleza independientemente de su utilidad y por otro lado, se estableció una reserva constitucional para el establecimiento de estos derechos.

Según (Cornejo, 2014) el desarrollo evolutivo de los derechos como el reconocimiento de la titularidad de los derechos de la naturaleza es una etapa inevitable" haciendo alusión dentro de su ensayo Los derechos de la naturaleza fundamentos, la determinación de ciertos elementos como se resumen en la tabla $\mathrm{N}^{\circ} 1$, mostrada a continuación:

Tabla 1. Derechos de la Naturaleza

\begin{tabular}{|c|l|}
\hline \multicolumn{1}{|c|}{ Derecho } & \multicolumn{1}{c|}{ Reconocimiento } \\
\hline \hline Dignidad & $\begin{array}{l}\text { La naturaleza y el ser humano siempre } \\
\text { serán un medio, que necesitan el uno del } \\
\text { otro para vivir. }\end{array}$ \\
\hline Derecho subjetivo & $\begin{array}{l}\text { Protección de los seres humanos y de la } \\
\text { naturaleza, como sujetos amparados dentro } \\
\text { de un estado constitucional. }\end{array}$ \\
\hline Capacidad & $\begin{array}{l}\text { Vinculada con la libertad, y el concepto de } \\
\text { titularidad de derechos para vender, obrar, } \\
\text { contratar, decidir, ejercer funciones y } \\
\text { contraer obligaciones. }\end{array}$ \\
\hline Igualdad & $\begin{array}{l}\text { Entendida como un contrato que incluye la } \\
\text { participación con representación de seres } \\
\text { no humanos como la naturaleza. }\end{array}$ \\
\hline Los animales tengan un lugar intermedio \\
entre los seres humanos y las cosas, \\
entendiéndose que son entes capaces de
\end{tabular}




\section{Derechos de la naturaleza frente a los delitos ambientales provocados por las actividades antropogénicas en la provincia de Los Ríos}

Vol. 3, núm. 3 Esp., (2019) Roberto Johan Barragán Monrroy; Johana Narcisa Ormaza Varela; Gissela Eloísa Vargas Cedeño; Gabriela Verónica Estrella Bravo

\section{sufrir y de sentir.}

Fuente: Elaboración propia.

\section{Delitos ambientales}

Es aquella conducta típica, antijurídica y culpable, sancionada con una pena como consecuencia de realizar actos que contravienen disposiciones administrativas, normativas y reglamentarias que causan un daño al medio ambiente. Se define como un crimen contra el medio ambiente que es sancionado por la legislación medioambiental. Es una infracción contra la legislación medioambiental, cuya sanción judicial está clasificada en la categoría de crimen. Se fundamenta en el deber de todos y cada uno de participar en la protección del medio ambiente, entendido como el bien común que debe ser preservado (Tello, 2015).

\section{Los tipos penales contenidos en el Código Orgánico Integral Penal (COIP)}

En el Código Orgánico Integral Penal se encuentran tipificados los delitos ambientales y la forma de juzgar y sancionarlos; así como velar por que se repare integralmente a las víctimas del mismo. Es necesario para el derecho penal que las conductas punitivas se encuentren tipificadas en el COIP, se describirán brevemente los delitos establecidos en la norma penal que se encuentran tipificados actualmente como actividades antropogénicas:

El Art. 246 del COIP menciona: la persona que provoque directa o indirectamente incendios o instigue la comisión de tales actos, en bosques nativos o plantados o páramos, será sancionada con pena privativa de libertad de uno a tres años. Se exceptúan las quemas agrícolas o domésticas realizadas por las comunidades o pequeños agricultores dentro de su territorio. Si 


\section{Derechos de la naturaleza frente a los delitos ambientales provocados por las actividades antropogénicas en la provincia de Los Ríos}

Vol. 3, núm. 3 Esp., (2019)

Roberto Johan Barragán Monrroy; Johana Narcisa Ormaza Varela; Gissela Eloísa Vargas

Cedeño; Gabriela Verónica Estrella Bravo

estas quemas se vuelven incontrolables y causan incendios forestales, la persona será sancionada por delito culposo con pena privativa de libertad de tres a seis meses. Si como consecuencia de este delito se produce la muerte de una o más personas, se sancionará con pena privativa de libertad de trece a dieciséis años.

El Art. 247 del C.O.I.P. señala: la persona que cace, pesque, capture, recolecte, extraiga, tenga, transporte, trafique, se beneficie, permute o comercialice, especímenes o sus partes, sus elementos constitutivos, productos y derivados, de flora o fauna silvestre terrestre, marina o acuática, de especies amenazadas, en peligro de extinción y migratorias, listadas a nivel nacional por la Autoridad Ambiental Nacional así como instrumentos o tratados internacionales ratificados por el Estado, será sancionada con pena privativa de libertad de uno a tres años.

El Art. 251 del C.O.I.P. señala: la persona que, contraviniendo la normativa vigente, contamine, deseque o altere los cuerpos de agua, vertientes, fuentes, caudales ecológicos, aguas naturales afloradas o subterráneas de las cuencas hidrográficas y en general los recursos hidrobiológicos o realice descargas en el mar provocando daños graves, será sancionada con una pena privativa de libertad de tres a cinco años. Se impondrá el máximo de la pena si la infracción es perpetrada en un espacio del Sistema Nacional de Áreas Protegidas o si la infracción es perpetrada con ánimo de lucro o con métodos, instrumentos o medios que resulten en daños extensos y permanentes.

El Art. 253 del C.O.I.P. expresa: la persona que contamine el aire, la atmósfera o demás componentes del espacio aéreo en niveles tales que resulten daños graves a los recursos 


\section{Derechos de la naturaleza frente a los delitos ambientales provocados por las actividades antropogénicas en la provincia de Los Ríos}

Vol. 3, núm. 3 Esp., (2019) Roberto Johan Barragán Monrroy; Johana Narcisa Ormaza Varela; Gissela Eloísa Vargas

Cedeño; Gabriela Verónica Estrella Bravo

naturales, biodiversidad y salud humana, será sancionada con pena privativa de libertad de uno a tres años.

El Art. 260 del COIP expresa: la persona que, sin autorización de la autoridad competente, extraiga, explote, explore, aproveche, transforme, transporte, comercialice o almacene recursos mineros, será sancionada con pena privativa de libertad de cinco a siete años. En caso de minería artesanal será sancionada con pena privativa de libertad de uno a tres años. $\mathrm{Si}$ producto de este ilícito se ocasionan daños al ambiente, será sancionada con pena privativa de libertad de siete a diez años.

El Art. 266 del COIP. Señala: la persona que por medios fraudulentos o clandestinos se apodere de hidrocarburos, sus derivados, incluido el gas licuado de petróleo y biocombustibles, cuando sean transportados a través de un oleoducto, gasoducto, poliducto o a través de cualquier otro medio o cuando estos se encuentren almacenados en fuentes inmediatas de abastecimiento o plantas de bombeo, será sancionada con pena privativa de libertad de cinco a siete años.

\section{Efectos de las actividades antropogénicas}

Las actividades antropogénicas se definen como aquellas relacionadas con la influencia del hombre en la naturaleza. El término antropogénico se ha utilizado con énfasis sobre la cantidad de dióxido de carbono que se encuentra en la atmósfera, producida por las actividades humanas y con un gran efecto sobre el cambio climático contemporáneo. Existen diferentes tipos como por ejemplo la atmosférica, hídrica, de los suelos, radioactiva, visual, lumínica, acústica o 


\section{Derechos de la naturaleza frente a los delitos ambientales provocados por las actividades antropogénicas en la provincia de Los Ríos}

Vol. 3, núm. 3 Esp., (2019)

Roberto Johan Barragán Monrroy; Johana Narcisa Ormaza Varela; Gissela Eloísa Vargas

Cedeño; Gabriela Verónica Estrella Bravo

por basura (Fernandez, 2019). El cambio climático de origen antropogénico se caracteriza por la introducción de una gran cantidad energía en la atmósfera.

El suelo, el agua, los océanos, los bosques, la atmósfera y las especies están amenazados por la actividad humana y con ellos el bienestar de las personas. El cambio climático, la deforestación, la extinción de especies y el crecimiento de la población humana no han hecho más que agravar la situación. destacan una reducción del $26 \%$ en la cantidad de agua dulce disponible por habitante; una reducción en la captura de peces salvajes, a pesar de los esfuerzos; un aumento del 75\% en el número de zonas muertas en los océanos; una pérdida de más de 121 millones de hectáreas de bosque; continuos aumentos en las emisiones globales de carbono y en las temperaturas promedio; un aumento del 35\% de la población humana; y una reducción del $29 \%$ en el número de mamíferos, reptiles, anfibios, aves y peces (Ripple, 2017). Dentro de sus consecuencias destacan:

- La degradación del medio ambiente, ha perjudicado al planeta desde su aparición. La acción del hombre ha provocado una caída de la biodiversidad y la extinción de algunas especies. Los agentes contaminantes comunes y cotidianos son los desechos sólidos domésticos e industriales, exceso de fertilizante y productos químicos, los desagües de aguas negras al mar o ríos, el monóxido de carbono de los vehículos, etc.

- El calentamiento global es resultado del aumento de las concentraciones de dióxido de carbono en la atmósfera por la acción humana. A lo largo de los años se ha afectado a las tierras y su productividad, así como a los océanos y la capa de ozono. 


\section{Derechos de la naturaleza frente a los delitos ambientales provocados por las actividades antropogénicas en la provincia de Los Ríos}

Vol. 3, núm. 3 Esp., (2019)

Roberto Johan Barragán Monrroy; Johana Narcisa Ormaza Varela; Gissela Eloísa Vargas

Cedeño; Gabriela Verónica Estrella Bravo

- El ser humano ha sufrido las consecuencias de sus propias acciones. Se ha demostrado que el calentamiento global y la contaminación tienen efectos negativos en la salud de las personas.

Una mirada a las cifras

Un reporte publicado los primeros días de junio de 2014 por Naciones Unidas (NU) encontró que el valor de los delitos ambientales aumentó ese año en un 26\% comparado con las estimaciones para ese período. Los crímenes contra la naturaleza, desde la pesca ilegal al tráfico de marfil, producen hasta USD 258000 millones anualmente, situándolos en la cuarta actividad ilegal más lucrativa del mundo, por detrás del tráfico de drogas, falsificación y la trata humana. En el informe se destaca el caso del pequeño mamífero pangolín, como el animal más traficado del mundo; en Ecuador el mono ardilla es una de los favoritos para el tráfico de animales. Según el Ministerio del Ambiente (MAE), en el 2015 se traficaron 506 animales (Sorgato, 2016).

El crimen más evidente en el país es la contaminación de los ríos. No obstante, en el artículo 251 del Código Orgánico Integral Penal (COIP) se sanciona con pena privativa de libertad de 3 a 5 años, a quien contamine vertientes, fuentes y caudales. La tala ilegal es el delito más común a escala mundial. Según el MAE, de 1990 hasta el 2014 el Ecuador perdió 1,8 millones de hectáreas de bosque. En cuanto a la pesca ilegal, "Ecuador y Costa Rica son de los principales productores de aletas de tiburón, puntos de tránsito y transbordo para cocaína que ingresa a EE.UU. y a Europa”, según un informe del 2015 de la Fiscalía General del Estado (Sorgato, 2016). 


\section{Derechos de la naturaleza frente a los delitos ambientales provocados por las actividades antropogénicas en la provincia de Los Ríos}

Vol. 3, núm. 3 Esp., (2019)

Roberto Johan Barragán Monrroy; Johana Narcisa Ormaza Varela; Gissela Eloísa Vargas

Cedeño; Gabriela Verónica Estrella Bravo

Tabla 2. Resumen de problemas ambientales en Ecuador para el año 2017

El lunes 14 de agosto, Ecuador capturó en aguas de la sensible reserva de las islas Galápagos un buque de bandera china con unas 300 toneladas de pesca que incluyen al menos dos especies de tiburón consideradas vulnerables por las autoridades de Ecuador como tiburones martillo (en peligro de extinción) y silky. La noche del domingo 27 de agosto, los tripulantes del barco fueron sentenciados con prisión de entre uno y cuatro años y el pago de una elevada multa.

El 15 de noviembre se conoció que un derrame de hidrocarburos contaminó el río Teaone, ubicado en la parroquia Vuelta Larga, provincia de Esmeraldas, y la mancha se expandió hasta el río Esmeraldas. Según detallaron las autoridades, el hecho se habría originado por una falla operativa en las válvulas de aceite quemado de la empresa pública Termoesmeraldas.
La tala de al menos 30 cipreses en la avenida Chirijos, en Milagro, motivó el reclamo de personas los primeros días de noviembre. La alcaldesa de Milagro argumento que la orden que emitió fue la de retirar y replantar los árboles en un lugar cercano al cantón, pero que los funcionarios se tomaron atribuciones que no les correspondían y los talaron.

Un derrame de petróleo afectó a una laguna de aproximadamente 120 metros de longitud y entre 5 y 20 metros de ancho, en Sucumbíos. En el sector de Campo Bello 2, pobladores reportaron una fuga el sábado 2 de diciembre en una tubería que transporta crudo de un pozo petrolero hacia la estación Atacapi, operada por la estatal Petroamazonas.

Fuente: (Gómez, 2017).

La crisis ambiental en muchos casos es severa, no existen métodos detallados para medirla cabalmente, hay evidencia suficiente que afecta al desarrollo de países y sociedades, tanto ricos, como pobres. El incremento del desarrollo está bajo amenaza a nivel global debido al aumento del riesgo de desastres. En algunos países, el riesgo de perder riqueza por los desastres supera la tasa de creación de riqueza (CEPAL, 2017). 


\section{Derechos de la naturaleza frente a los delitos ambientales provocados por las actividades antropogénicas en la provincia de Los Ríos}

Vol. 3, núm. 3 Esp., (2019) Roberto Johan Barragán Monrroy; Johana Narcisa Ormaza Varela; Gissela Eloísa Vargas

Cedeño; Gabriela Verónica Estrella Bravo

Las consecuencias ambientales negativas suelen afectar más a las personas más pobres que dependen directamente de los recursos naturales para su supervivencia. Además, hay que considerar, que al menos $40 \%$ de todos los conflictos internos de los países de los últimos 60 años han tenido un vínculo con los recursos naturales (CEPAL, 2017), que enfrentan la depredación y la tensión entre explotación de recursos no renovables y renovables. La extinción de especies se debe a múltiples factores, el mayor, seguramente es la pérdida de hábitat, pero también se conjugan presiones directas como la sobreexplotación y el comercio legal e ilegal que tienen un impacto enorme en ciertos grupos de especies, especialmente carismáticas como cactos, orquídeas o aves vistosas y también aquéllas usadas para alimento.

\section{Conclusiones.}

La debilidad de ciertas leyes y la falta de financiamiento en seguridad han permitido que las redes criminales y los grupos armados se beneficien del comercio de animales en número crítico y otros regalos de la naturaleza como minerales preciosos, alimentando los conflictos, devastando ecosistemas y amenazando a las especies en extinción, son actividades muy lucrativas que en nada consideran el precio que pagaran generaciones futuras por la destrucción del medio ambiente.

El Derecho es el instrumento apropiado, aunque no el único, para establecer mecanismos de delimitación en conflicto y de protección del interés del colectivo. Estableciendo expresamente el mandato de medidas penales para garantizar la protección ambiental y responder a la necesidad de dar una respuesta contundente a las intolerables agresiones que sufre el medio ambiente. La lista de delitos en contra de la biodiversidad tipificados y sancionados por el 


\section{Derechos de la naturaleza frente a los delitos ambientales provocados por las actividades antropogénicas en la provincia de Los Ríos}

Vol. 3, núm. 3 Esp., (2019)

Roberto Johan Barragán Monrroy; Johana Narcisa Ormaza Varela; Gissela Eloísa Vargas

Cedeño; Gabriela Verónica Estrella Bravo

Código Orgánico Integral Penal se evidencia que los legisladores quedaron en deuda con la población ecuatoriana, en relación a proteger al conjunto de flora y fauna que se encuentra en los diferentes ecosistemas ya indicados, para garantizar la diversidad dentro de cada especie en relación con las otras que comparten un mismo nicho ecológico o un mismo ecosistema.

Se han evidenciado algunos logros en término de actuación de funcionarios y aplicación de las Leyes para disminuir las actividades antropogénicas, pero no es suficiente. Las Leyes por si solas no erradicaran estas prácticas delictivas sin la educación del colectivo, para contrarrestar estos hechos es importante concientizar al ciudadano de su responsabilidad en la merma de la calidad del ambiente, es un costo que debe evaluar más allá de una ganancia monetaria.

Se trata de preservar su calidad de vida y por ende la del ambiente, mientras peor se trate el suelo con químicos, menor será la calidad del cultivo y el rendimiento de las actividades agrícolas, la contaminación del agua por desechos y petróleo no solo compromete la frescura del agua sino también muchas especies marinas cuyos ríos y mares son su habitad. Las consecuencias ambientales negativas suelen afectar más a las personas más pobres que dependen directamente de los recursos naturales para su supervivencia.

\section{Bibliografía.}

Bedón, R. (Abril 2017). Aplicación de los derechos de la naturaleza en Ecuador. Revista Veredas do Direito, Belo Horizonte, 14(28), 103.

CEPAL. (Mayo de 2017). Daño y pérdida de biodiversidad. Obtenido de CEPAL: www.cepal.org

Cornejo, J. (2014). Derechos de la naturaleza. Obtenido de Derecho Ecuador: https://www.derechoecuador.com 


\section{Derechos de la naturaleza frente a los delitos ambientales provocados por las actividades antropogénicas en la provincia de Los Ríos}

Vol. 3, núm. 3 Esp., (2019) Roberto Johan Barragán Monrroy; Johana Narcisa Ormaza Varela; Gissela Eloísa Vargas Cedeño; Gabriela Verónica Estrella Bravo

Fernandez, A. (Junio de 2019). Actividades antropogénicas: origen, características, consecuencias. Obtenido de Lifeder: https://www.lifeder.com

Gómez, V. (13 de Diciembre de 2017). Delitos ambientales en Ecuador registrados durante el 2017. El Universo, pág. https://www.eluniverso.com.

Gudynas, E. (17 de Abril de 2009). Derechos de la naturaleza en Ecuador. Obtenido de Acción $\mathrm{y}$ reacción: http://accionyreaccion.com

Martínez, A. (2019). El nuvo marco jurídico ambiental en Ecuador. Estudio sobre el código orgánico del ambiente. Actualidad Jurídica Ambiental(89), 1-32.

Ripple, W. (13 de Noviembre de 2017). Las actividades antropogénicas perjudican seriamente al planeta. Obtenido de TYS Magazine: https://www.tysmagazine.com

Sorgato, V. (12 de Junio de 2016). Acelerado daño de la tierra por los delitos ambientales. Obtenido de El Comercio: https://www.elcomercio.com

Tello, J. (Febrero de 2015). Los Delitos Ambientales en la Legislación Ecuatoriana vigente. Quito, Ecuador.

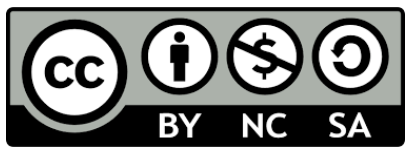

RECONOCIMIENTO-NOCOMERCIAL-COMPARTIRIGUAL

CC BY-NC-SA

ESTA LICENCIA PERMITE A OTROS ENTREMEZCLAR, AJUSTAR Y CONSTRUIR A PARTIR DE SU OBRA CON FINES NO

COMERCIALES, SIEMPRE Y CUANDO LE RECONOZCAN LA AUTORÍA Y SUS NUEVAS CREACIONES ESTÉN BAJO UNA LICENCIA CON LOS MISMOS TÉRMINOS. 